\title{
INFLUENCE OF SKIN EFFECT ON THE CALCULATION OF BUSBAR SYSTEMS HAVING RECTANGULAR CROSS SECTION
}

\author{
UDC (639.1.081.12:(669.15:620.193.4))
}

\section{Nebojša Raičević, Ana Vučković, Mirjana Perić, Slavoljub Aleksić}

University of Niš, Faculty of Electronic Engineering, Department of Theoretical Electrical Engineering, Republic of Serbia

\begin{abstract}
One method for the calculation of current density distribution in a finite number of long parallel conductors, having rectangular cross section, is proposed in this paper. Numerical results aim to highlight the importance of the skin effect, which can be combined with the proximity effect. The method of superposition of these two effects was applied to the calculation of the electromagnetic field in electric power busbars systems. It has been shown that the skin effect has a much greater impact, especially when the conductors are thin and strong electric currents flow through them, so special attention is paid to its calculation. For numerical solution the integral equations are used. The function of current density is approximated by the finite functional series. This way leads to a very accurate solution with only two terms. Differential evolution method is applied for minimization of error function. To demonstrate the application of the proposed approach, numerical values for busbars are presented and compared with values obtained by using the finite elements method.
\end{abstract}

Key words: Busbar, skin effect, differential evolution method, iterative method, current density distribution

\section{INTRODUCTION}

The skin effect is the appearance of a non-uniform distribution of alternating current across the cross section of the conductor. The current density is greatest at the surface of the conductor and decreases inside the conductor, so the skin effect is the result of the alternating current tendency to be distributed inside the conductor. This effect is more pronounced the thicker the conductor and the higher the frequency. High currents conductors have large cross sections and the current density is not uniform in them even

Received January 18, 2021 / Accepted February 17, 2021

Corresponding author: Nebojša Raičević

University of Niš, Faculty of Electronic Engineering, Aleksandra Medvedeva 14, 18000 Niš, Republic of Serbia

E-mail: nebojsa.raicevic@elfak.ni.ac.rs 
at low frequencies. The electric current flows mainly on the surface of the conductor, between the outer surface and the level called the depth of the conductor.

The skin effect is more pronounced if the specific conductivity $(\sigma)$ of the conductor and its permeability $(\mu)$ are higher.

If there are two conductors at a short distance, then the time-varying current in one conductor affects the current distribution in the other. This effect is called the proximity effect.

If the currents in the conductors are in opposite directions, then the current density increases in the parts of the conductors that face each other. In this case, as with the skin effect, there is an increase in the resistance and Joule losses in the conductors.

Except in a small number of cases when the observed system has certain symmetry (circular conductor, coaxial line, strip line, split strip type conductors), the solution of the skin effect cannot be obtained analytically, so standard approximate numerical methods are used to solve the corresponding differential and integral equations.

When determining the unknown function of the current density distribution in the cross section of conductors, the following assumptions are introduced:

- the medium is homogeneous, linear and isotropic,

- the supply is sinusoidal,

- there is no propagation in the axial direction, and

- Conductions dominate over dielectric displacement currents.

The study of the skin effect is based on Maxwell's equations and the concept of the macroscopic electromagnetic field. Starting from Maxwell's equations, neglecting displacement currents the Helmholtz equations can be obtained. The numerical method [1] that consisted of consecutive approximations for function of current density use normal models. The method itself consists of integral equation solving, where the unknown current density is presented in the shape of two-dimensional finite power with unknown complex coefficients.

One method for calculating the self-inductance of an isolated conductor, having rectangular cross section is presented in [2]. It defines the self-inductance of conductor of any shape and finite length given by multiple definite integral of six variables.

Method of moments can be applied on high frequencies to integral equations for computing internal impedance of a rectangular conductor, where the cross section is discretized to improve the convergence, by a non-uniform grid that obeys the skin effect [3].

In contrast with other methods, current density function can be approximated [4] by finite functional series which automatically satisfy wave equation. The Least Square Method (LSM) and Differential Evolution Method (DEM), one variant of the meta-heuristic optimization method (MHOM), are applied in order to minimize functional.

An approximate, but very simple and accurate method for calculating the current density distribution, skin-effect resistance ratio and ratio of inductance for alternating and direct current, in the pulse regime of a cylindrical conductor, having circular or rectangular crosssection, is presented in [5]. Direct and inverse Fast Furrier's transform is applied. As a very interesting in the practice, the lightning current pulse is observed.

It is possible to find an analytical-numerical method for the skin and proximity effects for a system of two parallel conductors of circular cross section in literature [6]. The magnetic field generated by the current applied on each conductor is expressed by means of the vector magnetic potential and expanded into Fourier series. Using the Laplace and Helmholtz equations, as well as the classical boundary conditions, the current density induced due to the 
proximity and the skin effect is determined in each conductor. The resulting current density is expressed as a series of successive reactions.

Also, the analytical-numerical approach to the skin and proximity effect in line with round parallel wires is proposed and based on the method of successive reactions [7]. The solution with substitutive filaments is considered as the first approximation of the true solution. Singlephase communication lines or three-phase bus ducts are considered to detect the limits of applicability of the first approximation. It has been shown that the error of the first approximation grows with wire radius to skin depth ratio and wire radius to wire spacing ratio. When the wire radius to skin depth ratio is up to 1 , and the gap between the wires is above the wire radius, the error is at a level of $1 \%$. However, lowering the distance and/or skin depth leads to a much larger error in the first approximation.

Bubnov-Galerkin method could be applied to determine the electromagnetic field of a system consisting of two conductors with rectangular cross-section, carrying identical alternating currents [8].

Numerical and analytical approaches for the study of busbar systems are analysed and compared [9]. Experimental measurements of the LF skin effect are given for a number of copper wires with different diameters and various linear solid cylindrical conductors including rods of copper, aluminium and brass [10].

The basic idea of this paper is to improve the accuracy and simplify the calculation of the skin effect in systems of parallel conductors, which have a rectangular cross section. It is supposed that every conductor has a calculated current density distribution. That would be the $0^{\text {th }}$, i.e. the initial approximation $[6,7]$. This current generates a time harmonic magnetic field which induces eddy currents in the other conductors, and affects the current distribution in the cross section of another rectangular conductor. This current is a secondary source of magnetic field which in turn induces eddy currents in every conductor...

The first correction was made due to the proximity effect. Due to the shape and dimensions of rectangular conductors, as well as due to their mutual positions, this correction can be neglected in electric power busbars [11].

\section{SKIN EFFECT DETERMINATION}

The classical skin effect is a well-known phenomenon. Numerous publications describe analytical methods to calculate the influence of the skin effect for arrangements of cylindrical conductors in free space. In the literature, there are two classes of techniques to calculate electromagnetic field distribution in conductors: volumetric and surface methods. Volumetric techniques based on the Finite Element Method, or volumetric integral equations require a fine discretization of the entire cross-section of the conductor in order to capture the skin effect. Such techniques lead to a large number of unknowns and long computation times.

Finding closed exact analytical forms is possible only in special cases with axial symmetry. Other systems require a numerical approach.

This approach is based on simplification and treats other conductors as thin strips, neglecting the finite dimensions of the cross-section of the other conductors.

\subsection{System of isolated cylindrical conductors having arbitrary cross-section}

In order to obtain the integral equation, a current contour formed by a straight cylindrical infinitely long conductor of arbitrary but known cross section and a sufficiently 
far return conductor, also infinitely long, will be observed. Such two conductors connect at infinity, forming a closed contour (Fig.1.).

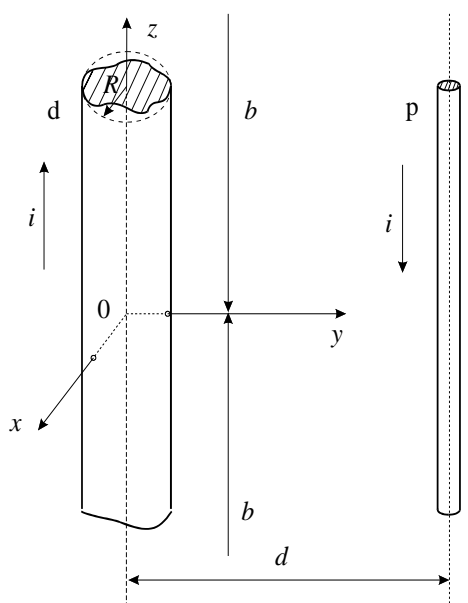

Fig. 1 Isolated cylindrical conductors

Given the described geometry of the problem, it is not difficult to conclude that the magnetic vector potential has a component composed of the part provided by the current of the direct conductor $(\mathrm{d})$, and the part provided by the current of the return conductor $(\mathrm{p})$ :

$$
A(\mathrm{x}, \mathrm{y})=A_{\mathrm{d}}(\mathrm{x}, \mathrm{y})+A_{\mathrm{p}}(\mathrm{x}, \mathrm{y})
$$

where

$$
A_{\mathrm{d}}(x, y)=\frac{\mu_{0}}{4 \pi} \int_{V_{\mathrm{d}}} \frac{J_{\mathrm{d}}\left(x^{\prime}, y^{\prime}\right) \mathrm{d} x^{\prime} \mathrm{d} y^{\prime} \mathrm{d} z^{\prime}}{\sqrt{\left(x-x^{\prime}\right)^{2}+\left(y-y^{\prime}\right)^{2}+z^{\prime 2}}},
$$

and

$$
A_{\mathrm{p}}(x, y)=-\frac{\mu_{0}}{4 \pi} \int_{V_{\mathrm{p}}} \frac{J_{\mathrm{p}}\left(x^{\prime}, y^{\prime}\right) \mathrm{d} x^{\prime} \mathrm{d} y^{\prime} \mathrm{d} z^{\prime}}{\sqrt{\left(x-x^{\prime}\right)^{2}+\left(y-y^{\prime}\right)^{2}+z^{\prime 2}}} .
$$

After the mentioned assumptions are included in the calculation, for the magnetic potential vector is obtained:

$$
A(x, y) \approx \frac{\mu_{0}}{2 \pi} i \ln d-\frac{\mu_{0}}{4 \pi} \int_{\mathrm{S}_{\mathrm{d}}} J_{\mathrm{d}}\left(x^{\prime}, y^{\prime}\right) \ln \left[\left(x-x^{\prime}\right)^{2}+\left(y-y^{\prime}\right)^{2}\right] \mathrm{d} x^{\prime} \mathrm{d} y^{\prime}+\frac{\mu_{0}}{2 \pi} i \ln (2 b) .
$$

The electric field vector is expressed through the electric scalar and the magnetic potential vector:

$$
\underline{\vec{E}}=-\operatorname{grad} \underline{\varphi}-j \omega \underline{\vec{A}}
$$

Having in mind all the above, we can finally write an integral equation for the current density of a direct conductor in the form: 


$$
J(x, y)=-\frac{k^{2}}{4 \pi} \int_{S} J\left(x^{\prime}, y^{\prime}\right) \ln \left[\left(x-x^{\prime}\right)^{2}+\left(y-y^{\prime}\right)^{2}\right] \mathrm{d} x^{\prime} \mathrm{d} y^{\prime}+\underline{C} .
$$

The obtained integral equation is of the Fredholm type.

For any conductor, neglecting the dimensions of neighboring wires, the skin-effect is given by integral equation:

$$
J(\vec{r})=J_{0}-\frac{k^{2}}{2 \pi} \int_{S} J\left(\vec{r}^{\prime}\right) \ln \left|\vec{r}-\vec{r}^{\prime}\right| \mathrm{d} S^{\prime},
$$

where $k^{2}=-\mathrm{j} \omega \mu \sigma, \mathrm{j}=\sqrt{-1}, J(\vec{r})$ - axial component of current density and $J_{0}=J(\vec{r})=C^{t e}$ for $\omega=0$. S is surface of conductor cross-section inside contour $C$.

Qualitatively, the preceding consideration hold for massive conductors of any crosssection with a smooth boundary, the only difference being that the tendency of the current density to concentrate towards the surface is more marked at the points where the curvature is greatest. It is possible to use $2 \mathrm{D}$ calculations in the cross-section of the configuration.

Isolated cylindrical conductors having arbitrary cross-section is shown in Fig.2.

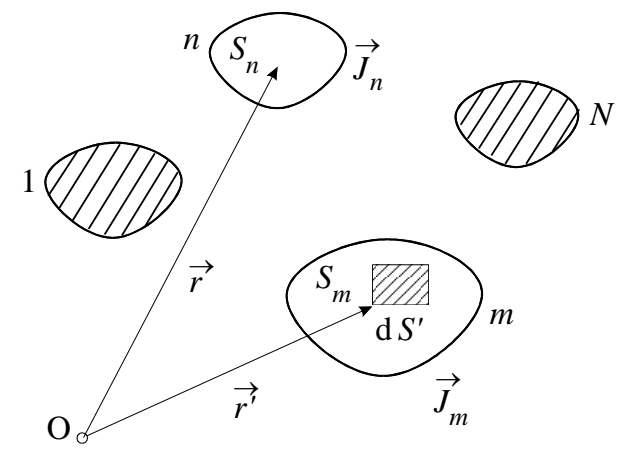

Fig. 2 Cross-section of conductors having arbitrary shape

The skin effect integral equation includes the wave equation:

$$
\Delta_{\mathrm{t}} J+k^{2} J=0,
$$

and boundary conditions. $\Delta_{\mathrm{t}}$ is transversal Laplacian.

The skin-effect is given by the integral equation:

$$
J_{n}(\vec{r})=J_{0 n}-\frac{k^{2}}{2 \pi} \sum_{m=1}^{N} \int_{S_{m}} J_{m}\left(\vec{r}^{\prime}\right) \ln \left|\vec{r}-\vec{r}^{\prime}\right| \mathrm{d} S^{\prime}, \quad n=1,2, \ldots, N,
$$

where $J_{n}(\vec{r})$ - axial component of current density for $n$-th conductor and $J_{0 n}=J_{0}(\vec{r})=C^{t e}$ for $\omega=0 . S$ is the surface of the n-th conductor cross-section.

The presented integral equation cannot be exactly analytically solved and that is why its approximate numerical solution is used. An analytical solution is known only for very simple cases as half space, infinite layer or the infinite conductor of circular cross-section. 


\subsection{System of isolated cylindrical conductors having rectangular cross-section}

The cylindrical conductor of rectangular cross-section is shown in Fig.2.

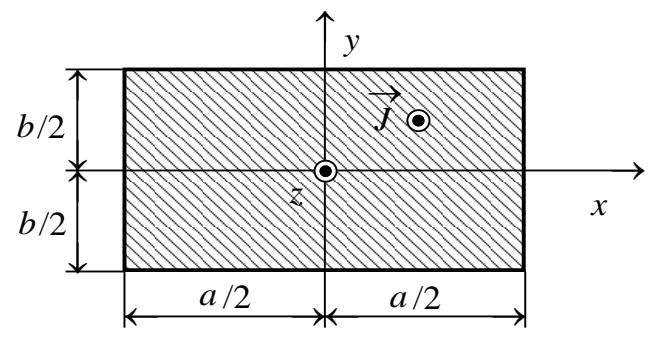

Fig. 3 Cross-section of conductor having rectangular shape

An iterative method can be successfully used to solve the derived differential and integral equations. The solution for the current density function is assumed in the form of the following unlimited order:

$$
J_{n}(\vec{r})=\sum_{v=0}^{\infty} k^{2 v} F_{n, 2 v}(\vec{r}), \quad n=1,2, \ldots, N
$$

The current density function is approximated by finite functional series which automatically satisfy the wave equation:

$$
\frac{\partial^{2} J}{\partial x^{2}}+\frac{\partial^{2} J}{\partial y^{2}}+k^{2} J=0
$$

and existing boundary conditions.

Solution of (10) is:

$$
F_{n, 2 v+2}(\vec{r})=-\frac{1}{2 \pi} \sum_{m=1}^{N} \int_{S_{m}} F_{m, 2 v}\left(\vec{r}^{\prime}\right) \ln \left|\vec{r}-\vec{r}^{\prime}\right| \mathrm{d} S^{\prime} .
$$

The current has only a z-component, but all components depend on the $\mathrm{x}$ and $\mathrm{y}$ coordinate. A separating variable method can be applied. Inserting

$$
J(x, y)=\sum_{n=0}^{\infty} X_{n}(x) \cos (n k y)
$$

in equation (11), the following expression for current density is obtained:

$$
J(x, y)=C_{0} \cos k x+C_{1} \cos k y+\sum_{n=2}^{\infty} C_{n} \cosh \left[k \sqrt{n^{2}-1} x\right] \cos n k y .
$$

In practice, it is usually necessary to cut the infinite series at certain terms. In the proposed method, it is sufficient to take into account only two terms:

$$
J(x, y)=C_{1} \cosh \left[k \sqrt{\alpha^{2}-1} x\right] \cos \alpha k y+C_{2} \cosh \left[k \sqrt{\alpha^{2}-1} y\right] \cos \alpha k x,
$$

where $\alpha$ is the parameter. $C_{1}, C_{2}$ and $\alpha$ are unknown values. 
The system of three equations is formed, two linear and the last one is non-linear, that is necessary to solve.

The Least Square Method (LSM) is combined with the Differential Evolution Method (DEM) in order to minimize functional:

$$
F=\sum_{i=1}^{I}\left\{C_{1}\left[A_{1 i}+\frac{k^{2}}{4 \pi} B_{1 i}\right]+C_{2}\left[A_{2 i}+\frac{k^{2}}{4 \pi} B_{2 i}\right]-J_{0}\right\}^{2}=\min ,
$$

where

$$
\begin{gathered}
A_{1 i}=A_{1}\left(x_{i}, y_{i}\right)=\cosh \left(k \sqrt{\alpha^{2}-1} x_{i}\right) \cos \left(\alpha k y_{i}\right), \\
B_{1 i}=B_{1}\left(x_{i}, y_{i}\right)=\int_{-a / 2}^{a / 2} \int_{-b / 2}^{b / 2} \operatorname{Ker} \mathrm{d} x^{\prime} \mathrm{d} y^{\prime}, \\
\operatorname{Ker}=\cosh \left(k \sqrt{\alpha^{2}-1} x^{\prime}\right) \cos \left(\alpha k y^{\prime}\right) \ln \left[\left(x_{i}-x^{\prime}\right)^{2}+\left(y_{i}-y^{\prime}\right)^{2}\right], \\
A_{2 i}=A_{2}\left(x_{i}, y_{i}\right)=A_{1}\left(y_{i}, x_{i}\right), \\
B_{2 i}=B_{2}\left(x_{i}, y_{i}\right)=B_{1}\left(y_{i}, x_{i}\right) .
\end{gathered}
$$

By differentiating of functional (16) according to $C_{1}, C_{2}$ and $\alpha$ the system of equations is obtained.

Results obtained after applying the DE method, one variant of the meta-heuristic optimization algorithm (MHOA) [12], are given in the last row. Meta-heuristics optimization is becoming a popular tool for solving numerous problems in electromagnetic field calculation due to the ability to overcome many shortcomings in traditional optimization. The metaheuristic algorithm is one that is designed to solve a problem in a faster and more efficient fashion than traditional methods with optimality and accuracy.

The optimization problem can be formulated in general form as:

$$
O F(\vec{x}) \rightarrow \min ,
$$

with the following restrictions:

$$
\begin{aligned}
& a_{L} \leq a_{i} \leq a_{U} \forall i \in(N=1, \ldots N), \\
& h_{L} \leq h_{i} \leq h_{U} \forall i \in(N=1, \ldots N),
\end{aligned}
$$

where $\vec{x}$ is the vector of the optimization problem decision variables.

The objective function for the optimization problem of optimal parameter values for calculation of the current distribution over the cross section of the rectangle conductor is defined by (16).

Because the parameters $C_{1}, C_{2}$ and $\alpha$ are complex numbers, the decision variable vector consists of the real and imaginary parts of the parameters:

$$
\vec{x}=\left[C_{1 R} C_{1 I} C_{2 R} C_{1 I} \alpha_{R} \alpha_{I}\right]^{\mathrm{T}} .
$$


The meta-heuristic optimization method Differential Evolution is used to solve the optimization problem. Because DE is very well here is given overview of the method in brief. The DE has similar structure as other Evolutionary Algorithms (EA) that can be presented as given in block diagram (Fig.4).

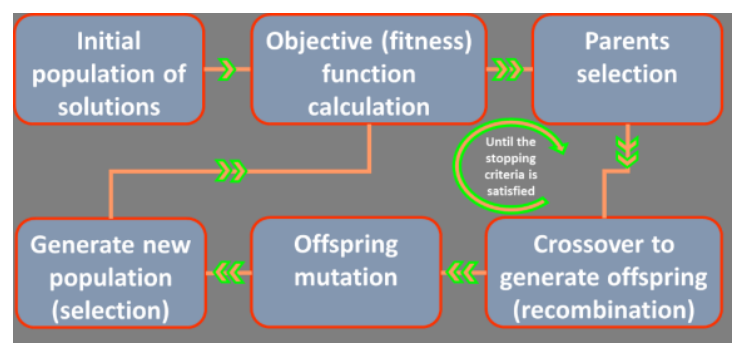

Fig. 4 Block diagram of Differential Evolution structure

The main operators in the EAs are mutation, crossover and selection.

The mutation in DE is done by calculation the mutant vector according to:

$$
\vec{x} m_{g}^{j}=\vec{x}_{g}^{r 1}+F \cdot\left(\vec{x}_{g}^{r 2}-\vec{x}_{g}^{r 3}\right)+F \cdot\left(\vec{x}_{g}^{r 4}-\vec{x}_{g}^{r 5}\right),
$$

for $r_{1} \neq r_{2} \neq r_{3} \neq r_{4} \neq r_{5}$, where coefficient $F$ is one of the DE parameters, called mutation factor in the range $[0,1]$.

The crossover procedure in the DE is done by using the trial vector. Based on the mutant vector the trial vector is defined with the elements:

$$
x t_{g, i}^{j}=\left\{\begin{array}{ll}
x m_{g, i}^{j} & \text { if }\left(\operatorname{rand}_{i}[0,1) \leq C_{r}\right) \\
x m_{g, i}^{j} & \text { otherwise }
\end{array},\right.
$$

The $C_{r}$ is a DE parameter, called the crossover rate in the range $[0,1]$. The selection in $\mathrm{DE}$ is defined by:

$$
\vec{x}_{g+1}^{j}=\left\{\begin{array}{ll}
\vec{x}_{g}^{j} & \text { if }\left(\operatorname{OF}\left(\vec{x} t_{g}^{j}\right) \leq \operatorname{OF}\left(\vec{x}_{g}^{j}\right)\right) \\
\vec{x}_{g}^{j} & \text { otherwise }
\end{array} .\right.
$$

The used optimization method is implemented in the Python programing environment using a built-in DE procedure from the SciPy Python package.

The presented method leads to a non-linear system of equations. After linearization, the convergence of the procedure is examined. The iterative procedure can be programmed relatively easily, since analytical formulae for coefficients can be derived.

The basic outputs of the program are numerical values of coefficients in analytical formulae that approximate the electromagnetic field in the conductor very well. The iterative procedure starts from $\alpha \rightarrow 0$ and after some iterations leads to the real value for the parameters $C_{1}, C_{2}$ and $\alpha$. The convergence of the procedure is presented in Table 1. 
Table $1 \alpha, C_{1}$ and $C_{2}$ convergency for recangular copper conductor $\left(\left(\mu=\mu_{0}\right), \sigma=57 \times\right.$ $10^{6} \mathrm{~s} / \mathrm{m}, a=b=8 \mathrm{~cm}$ ), on frequecy $f=50 \mathrm{~Hz}$

\begin{tabular}{ccc}
\hline $\begin{array}{c}\text { Number of } \\
\text { iterations }\end{array}$ & $\alpha$ & $C_{1}=C_{2}$ \\
\hline 2 & $0.15004-\mathrm{j} 0.00395$ & $0.03321-\mathrm{j} 0.02160$ \\
12 & $0.14673-\mathrm{j} 0.02464$ & $0.03531-\mathrm{j} 0.02372$ \\
20 & $0.13177-\mathrm{j} 0.03788$ & $0.03592-\mathrm{j} 0.02431$ \\
26 & $0.12102-\mathrm{j} 0.05001$ & $0.03610-\mathrm{j} 0.02433$ \\
30 & $0.12099-\mathrm{j} 0.05097$ & $0.03610-\mathrm{j} 0.02461$ \\
DEM & $\mathbf{0 . 1 2 0 0 3 - j 0 . 0 5 0 9 1}$ & $\mathbf{0 . 0 3 6 1 0 - j 0 . 0 2 4 6 6}$ \\
\hline
\end{tabular}

The intensity (absolute value) distribution of the current density, obtained by the proposed method for frequency $f=50 \mathrm{~Hz}$ and $J_{0}=1 \mathrm{~A} / \mathrm{m}^{2}$, in a quarter of the insulated conductor having square cross-section, the length of the side $a=8 \mathrm{~cm}$, is shown in Fig. 5 . Due to symmetry, the distribution of the current density is shown only in one-fourth of the cross-section of the conductor.

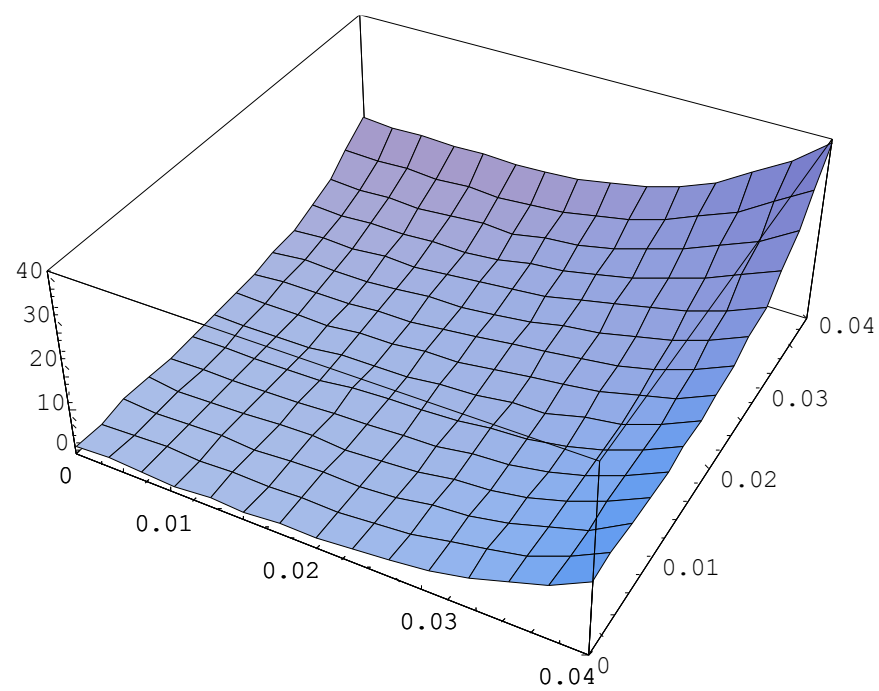

Fig. $53 \mathrm{D}$ distribution of intensity $\left[J_{0}=1 \mathrm{~A} / \mathrm{m}^{2}\right]$ of current density in insulated copper square conductor $(a=8 \mathrm{~cm}, f=50 \mathrm{~Hz})$

In the cross-sections are shown lines that connect points with the same current density magnitudes (Fig. 6). 


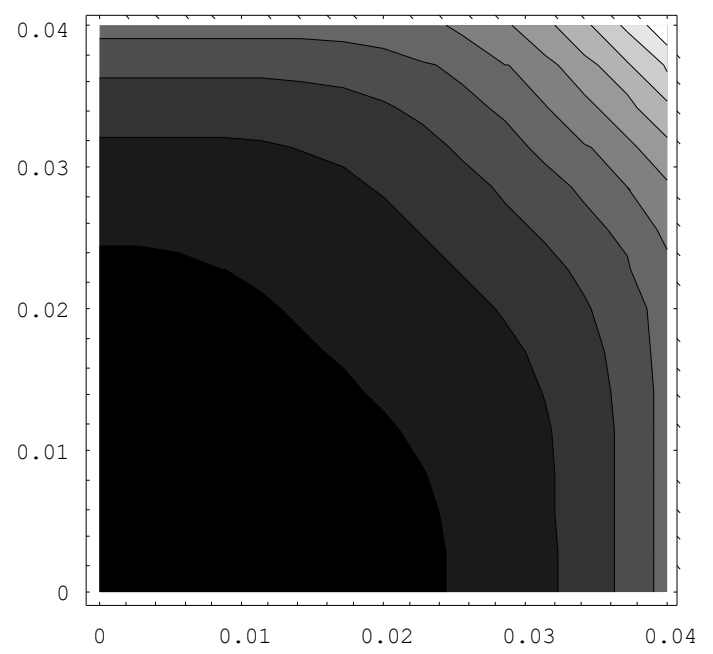

Fig. 6 Lines of constant magnetic field intensity $(\mathrm{mA} / \mathrm{m})$ for a square copper conductor $\left(a=8 \mathrm{~cm}, f=50 \mathrm{~Hz}\right.$ and $\left.J_{0}=1 \mathrm{~A} / \mathrm{m}^{2}\right)$

Lines of constant magnetic field intensity for a square copper conductor $(a=8 \mathrm{~cm}$, $f=50 \mathrm{~Hz}$ and $J_{0}=1 \mathrm{~A} / \mathrm{m}^{2}$ ) are presented in Fig. 7. Due to symmetry, the distribution of the magnetic field is shown only in one-fourth of the cross-section.

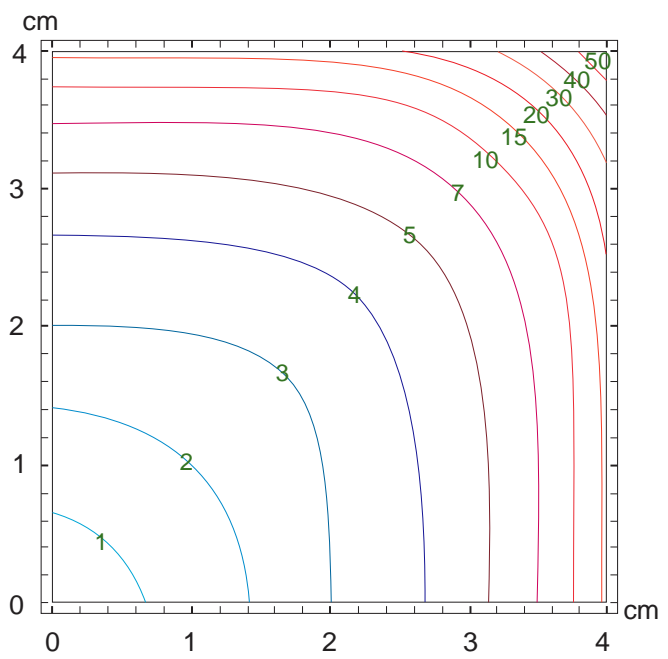

Fig. 7 Lines of constant magnetic field intensity $(\mathrm{mA} / \mathrm{m})$ for square copper conductor $\left(a=8 \mathrm{~cm}, f=50 \mathrm{~Hz}\right.$ and $\left.J_{0}=1 \mathrm{~A} / \mathrm{m}^{2}\right)$ 


\section{Calculation Of Busbar Systems Having Rectangular Cross Section}

The busbars are the electrical backbone of the switchgear. They connect the lines that supply energy to the lines that drain it. All lines are connected to the busbars, so they are of the utmost importance for the normal operation of the distribution plant. In the event of a bus fault, the relay protection must disconnect the busbars and all lines connected to them, which is a large power outage that can lead to severe consequences for the power system in high voltage plants.

High-current bus ducts with copper or aluminum rectangular busbars are often used in power substations and switching stations. In the case of a parallel conductor system, the distribution of current density in these conductors is caused by the skin and proximity effects, as well as the complete electromagnetic coupling between phase conductors and metal enclosures of the single-phase isolated bus duct system.

The skin effect is more noticeable in the square cross-section conductor $(a=b)$ than in the strips $(a>>b)$. Also, high power currents flow at power busbars, the conductors are distant, and so the proximity effect is smaller compared to the skin effect.

Let the conductor be in the form of a flat massive strip of infinity length, width $a$, and negligible thickness, $b(a>>b)$, as shown in Figure 8. The conductor is made of a material whose characteristics are $\varepsilon, \mu, \sigma$.

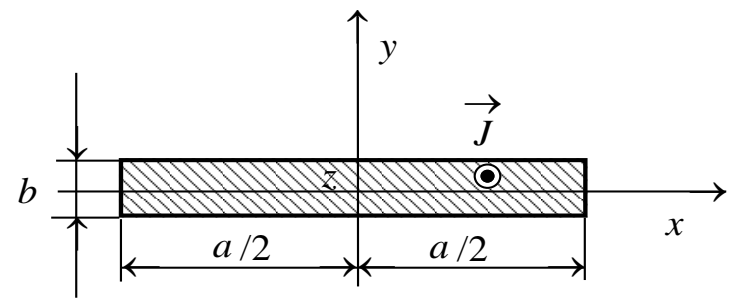

Fig. 8 Cross-section of conductor having rectangular shape $(a>>b)$

Integral equation for a flat massive strip is:

$$
J(x)=J_{0}-\frac{k^{2}}{4 \pi} \int_{-\mathrm{a} / 2}^{\mathrm{a} / 2} \int_{-\mathrm{b} / 2}^{\mathrm{b} / 2} J\left(x^{\prime}\right)\left[\ln \frac{\left(x-x^{\prime}\right)^{2}+y^{\prime 2}}{x^{\prime 2}+y^{\prime 2}}+\ln \frac{\left(x+x^{\prime}\right)^{2}+y^{\prime 2}}{x^{\prime 2}+y^{\prime 2}}\right] \mathrm{d} x^{\prime} \mathrm{d} y^{\prime},
$$

It is possible to apply in the point matching method. $N$ is the number of adjustment points.

Table 2 shows the convergence of the results for the ratio $J(x) / J(0)$ of a flat strip conductor, in the case when the skin effect is weak: $a / \delta=5.0(\delta=1 / \sqrt{\pi \mu \sigma f})$ and the degree of the polynomial is also different, $N=3,6,8$. In the same Table, in the last column, values of the same ratio obtained by using FEM are given. 
Table 2 Convergence of the results for the ratio $J(x) / J(0)$ of a flat strip conductor

\begin{tabular}{ccccc}
\hline $2 x / \mathrm{a}$ & $N=3$ & $N=6$ & $N=8$ & FEM \\
\hline 0.0 & 1.0000000 & 1.0000000 & 1.0000000 & 1.0000000 \\
0.1 & 1.0163064 & 1.0014676 & 1.0013006 & 1.0013013 \\
0.2 & 1.0664088 & 1.0206275 & 1.0206329 & 1.0206329 \\
0.3 & 1.1718596 & 1.1005219 & 1.1007146 & 1.1007149 \\
0.4 & 1.3544138 & 1.2934625 & 1.2934542 & 1.2934544 \\
0.5 & 1.6305333 & 1.6328639 & 1.6326567 & 1.6326582 \\
0.6 & 2.0263144 & 2.1304886 & 2.1304543 & 2.1304541 \\
0.7 & 2.5890609 & 2.7959696 & 2.7961016 & 2.7961016 \\
0.8 & 3.3845836 & 3.6506855 & 3.6506480 & 3.6506567 \\
0.9 & 4.4859109 & 4.7328712 & 4.7330578 & 4.7330394 \\
1.0 & 5.9639646 & 6.1032497 & 6.1030147 & 6.1030161 \\
\hline
\end{tabular}

It is necessary to check the magnetic field in busbars surroundings because the intensity must not exceed the maximum limited value [13]. This requires measurements around bus bar systems, but there is troublesome to measure magnetic fields in inaccessible places under the shield. Therefore, there are many numerical methods that deal with this issue and each contribution has great practical and theoretical significance.

The next numerical example selected for this paper features a three-phase system of rectangular horizontally placed busbars with one neutral conductor, whose cross-section is depicted in Fig. 9.

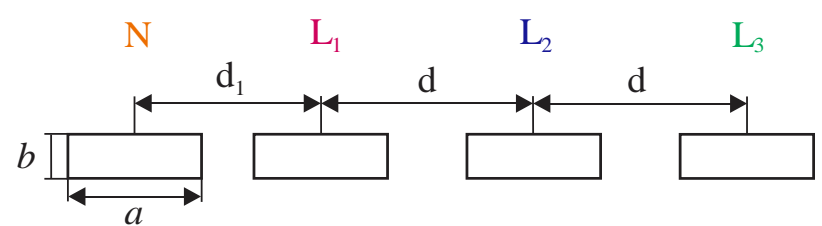

Fig. 9 Three-phase $\left(\mathrm{L}_{1}, \mathrm{~L}_{2}\right.$ and $\left.\mathrm{L}_{3}\right)$ high-current busbars having rectangular cross-section and one neutral busbar $(\mathrm{N})$

The distance between the bus conductors is defined by the voltage stresses in the power plant. According to the notations applied in this figure, the following geometry of the busbars has been selected: the dimensions of the phase rectangular busbars and the neutral busbars are $a=60 \mathrm{~mm}, b=5 \mathrm{~mm}, d=d_{1}=90 \mathrm{~mm}$. The phase busbars and the neutral are made of copper (Electrical conductivity $\sigma=59.6 \mathrm{MS} / \mathrm{m}$ ) and $f=50 \mathrm{~Hz}$. Because the skin-effect problem in thin conductors involves two dimensions of different orders of magnitude, a thickness $a$, and a lateral dimension $b$, with $b<<a$, it automatically splits into two almost unrelated problems.

Three balanced currents values $I_{\text {eff }}=1380 \mathrm{~A}$ are imposed in phases:

$$
I_{1}=I_{\text {eff }}, \quad I_{2}=I_{1} e^{-j \frac{2 \pi}{3}}, \quad I_{3}=I_{1} e^{j \frac{2 \pi}{3}} .
$$

Distribution of current and electromagnetic field in busbars can be successfully determined if the proximity effect is included together with skin effect in calculations.

Current density distribution (real part, imaginary part and magnitude) on the three busbar surfaces is given in Fig. 10, Fig. 11 and Fig. 12. Distribution along $x$ axes $(y=0)$ is presented in Fig. 13. 


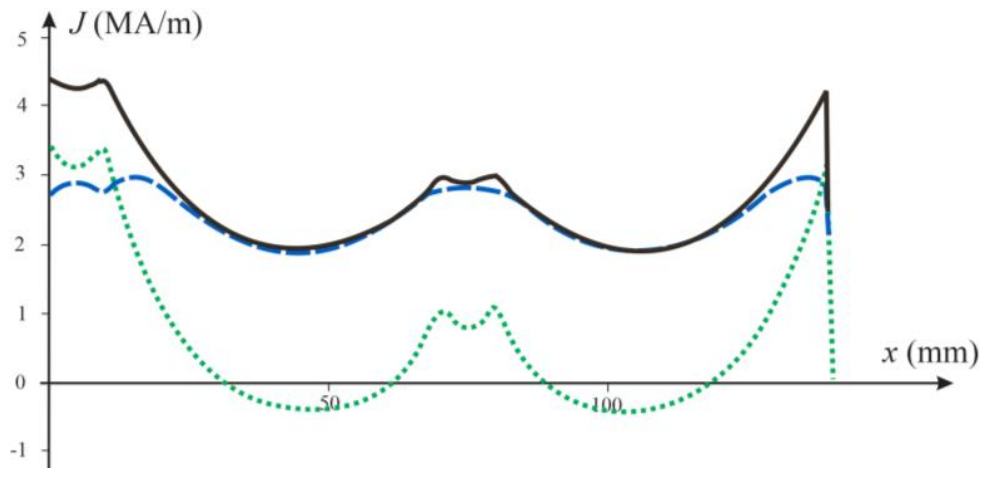

Fig. 10 Current density distribution on the first conductor $\left(L_{1}\right)$ surface (absolute value (solid line), real part (longer dashed line), Imaginary part (shorter dashed line))

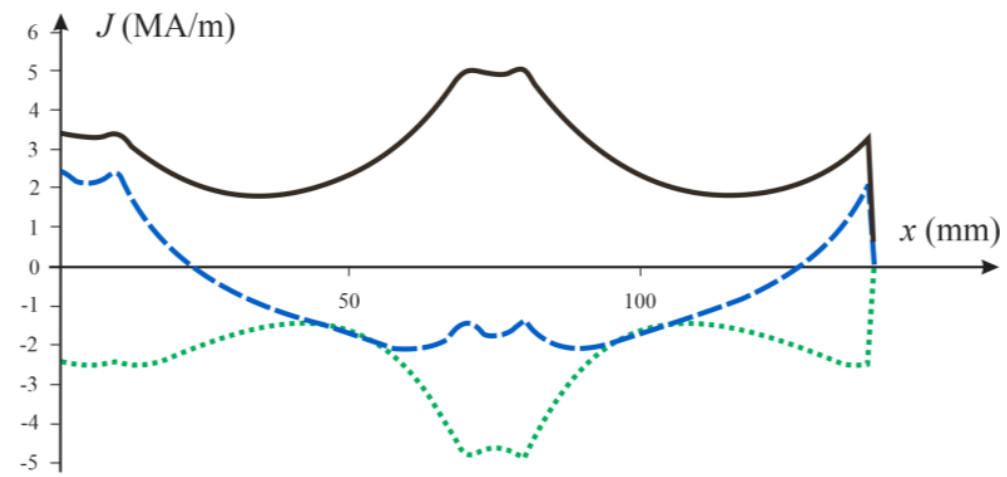

Fig. 11 Current density distribution on the second conductor $\left(L_{2}\right)$ surface (absolute value (solid line), real part (longer dashed line), Imaginary part (shorter dashed line))

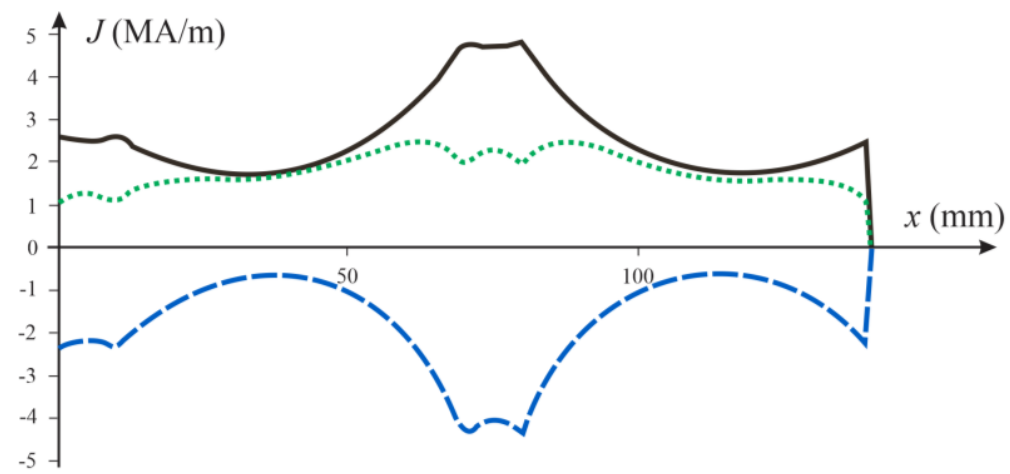

Fig. 12 Current density distribution on the third conductor $\left(L_{3}\right)$ surface (absolute value (solid line), real part (longer dashed line), Imaginary part (shorter dashed line)) 


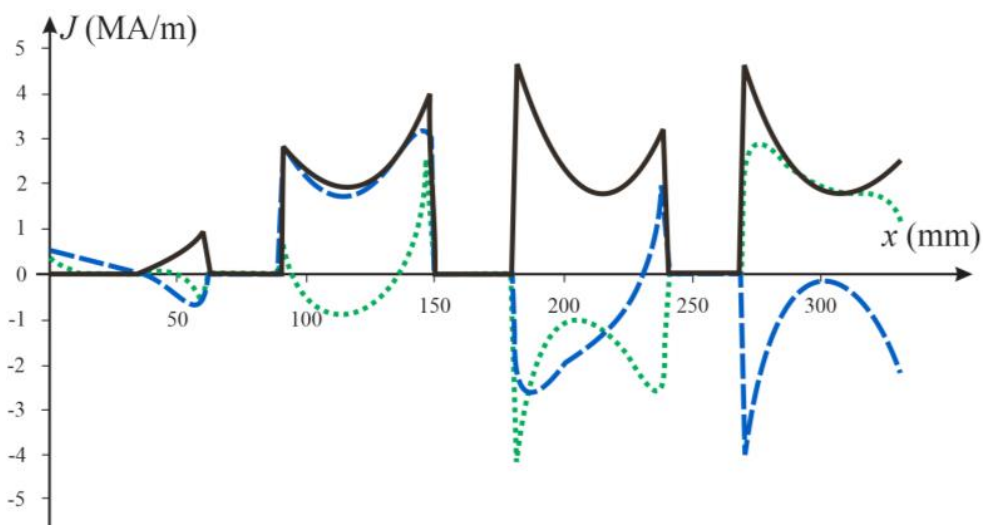

Fig. 13 Current density distribution along $x$ axes (absolute value (solid line), real part (longer dashed line), Imaginary part (shorter dashed line))

Magnetic field intensity along $x$ direction at points with different heights, in the highcurrent balanced three-phase busbars, having rectangular cross-section is shown in Fig. 14.

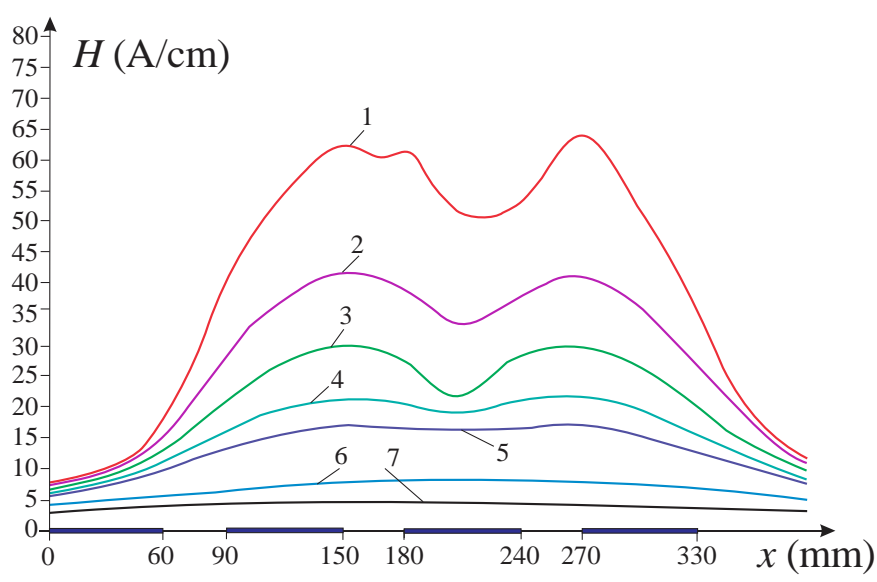

Fig. 14 Strength of magnetic field along $x$ direction at different heights $(y=-30 \mathrm{~mm}(3)$, $y=-15 \mathrm{~mm}(2), \quad y=0 \mathrm{~mm}(1), \quad y=45 \mathrm{~mm}(4), \quad y=75 \mathrm{~mm}(5), \quad y=145 \mathrm{~mm}(6)$, $y=210 \mathrm{~mm}(7))$ in the high-current balanced three-phase busbars, having rectangular cross-section ( $a=60 \mathrm{~mm}, b=5 \mathrm{~mm})$

The current density in busbars with rectangular conductors is not rotationally symmetric. Therefore, out-of-phase magnetic field components exist outside of the conductor.

The second configuration of a busbar system, the current density of which is investigated, is shown in Fig. 15. A system of $N$ rectangular vertically placed busbar's conductors is shown Fig. 16.

Fig. 16 refers to a group of five $80 \times 10 \mathrm{~mm}$ bars placed $10 \mathrm{~mm}$ apart. The curves drawn on the cross section are lines with the same current density. 


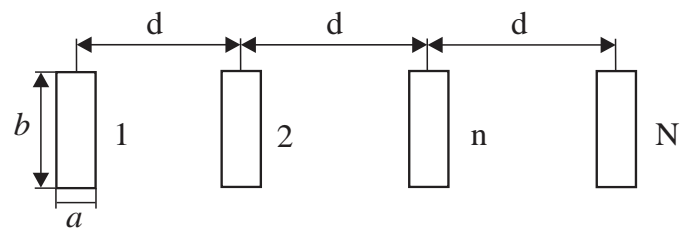

Fig. 15 Group of $\mathrm{N}$ bars having rectangular cross-section, placed at a distance $\mathrm{d}$

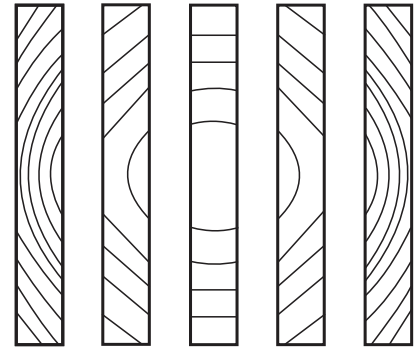

Fig. 16 Equi-current density lines for a group of of five 80 x $10 \mathrm{~mm}$ bars, placed $10 \mathrm{~mm}$ apart

It can be noticed that in a batch of neighboring bars the current distribution is only slightly different to that which would be produced merely by the skin effect in a solid conductor having the same external dimensions.

If currents are in the opposite direction, then the current density increases in the parts of the conductors facing each other. If the spacing between the busbars is from three to four times greater than their maximum dimensions, the proximity effect can be neglected.

The current density distribution (real part, imaginary part and magnitude) on the three busbar surfaces (Fig.15. for $N=3$ and when the conductors are supplied to the same as given by eq. (30)) is given in Fig. 17, Fig. 18 and Fig. 19. The distribution along $x$ axes $(y=0)$ is presented in Fig.20.

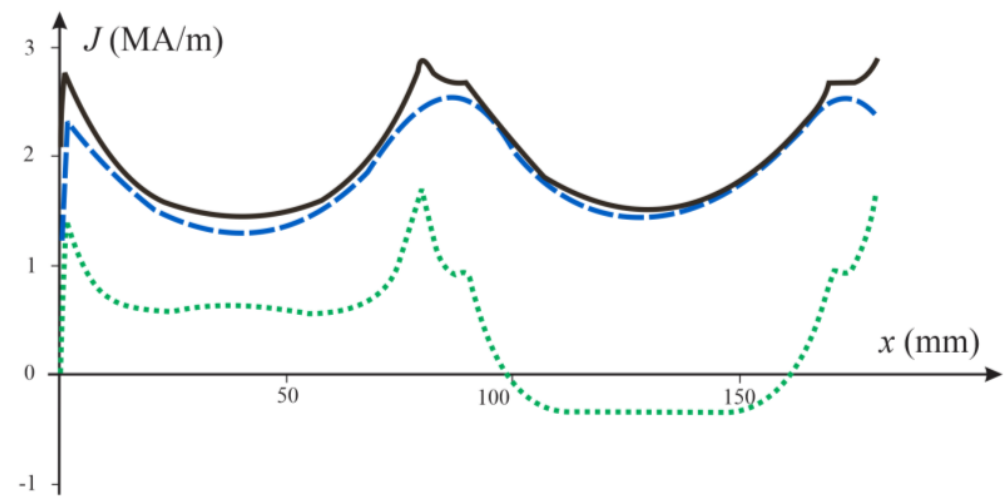

Fig. 17 Current density distribution on the first conductor (1) surface (absolute value (solid line), real part (longer dashed line), Imaginary part (shorter dashed line)) 


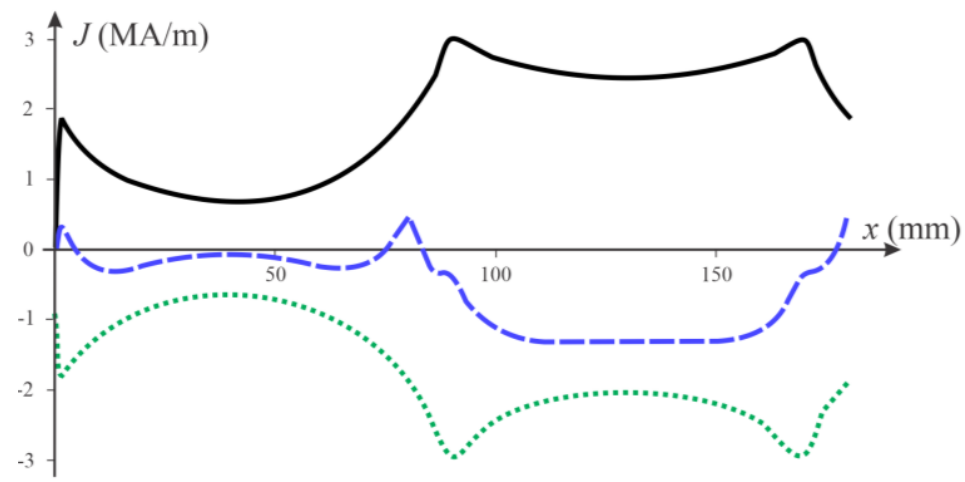

Fig. 18 Current density distribution on the first conductor (2) surface (absolute value (solid line), real part (longer dashed line), Imaginary part (shorter dashed line))

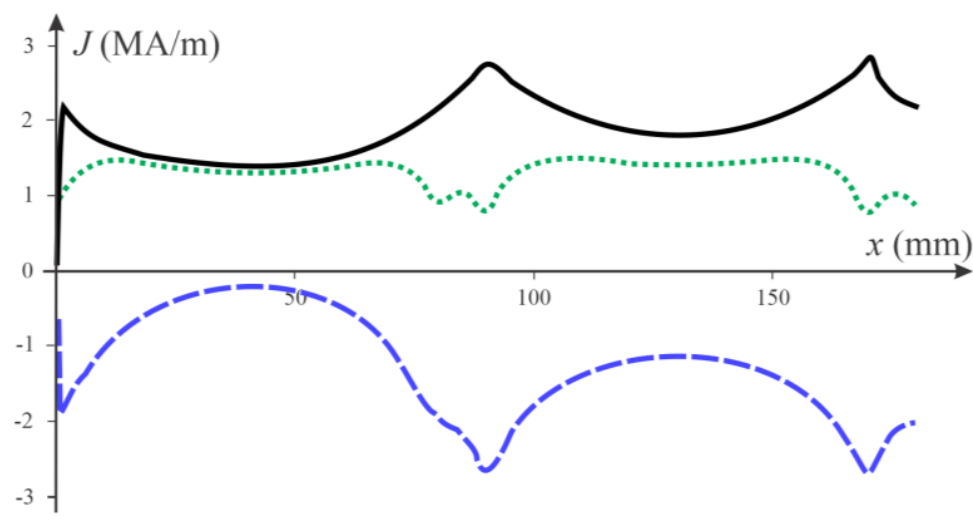

Fig. 19 Current density distribution on the first conductor (3) surface (absolute value (solid line), real part (longer dashed line), Imaginary part (shorter dashed line))

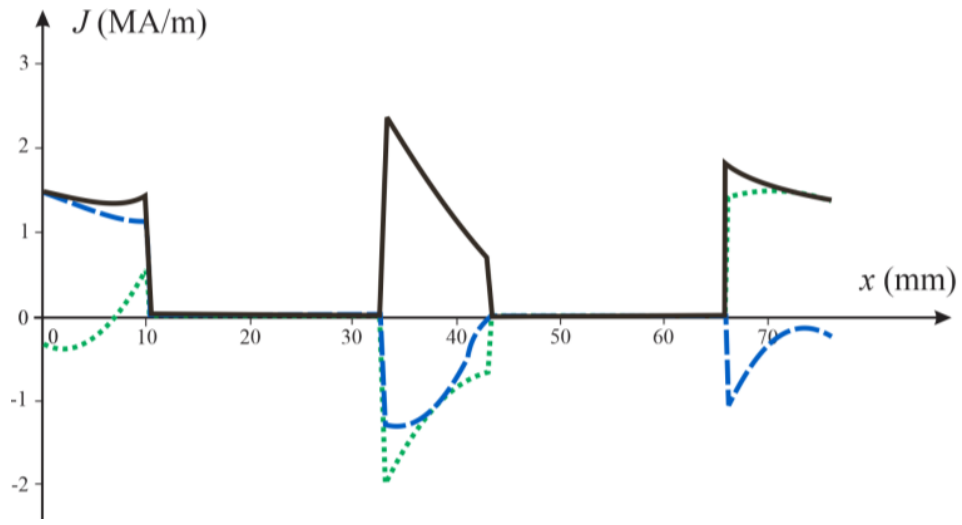

Fig. 20 Current density distribution along $x$ axes (absolute value (solid line), real part (longer dashed line), Imaginary part (shorter dashed line)) 


\section{CONCLUSION}

Based on the theory of electromagnetic fields and use of the integral equations, one simple method is proposed to determine the current density and the electromagnetic fields of rectangular busbar systems. This approach leads to a very accurate solution, despite the fact that only two terms in current approximation are adopted.

One simple method of calculating the current density in parallel conductors is suggested in this paper. The determined current densities in conductors, having rectangular cross section, can be used to calculate magnetic field, additional impedances, and power losses.

The considered problem of rectangular wires analyzed in this paper is still very attractive [14] and it is applicable in hybrid drives for passenger cars.

The results indicate that this numerical method can be used to predict the current density of any such rectangular busbar system with a good accuracy.

\section{REFERENCES}

[1] P. Silvester, "Modal Network Theory of Skin Effect in Flat Conductors," Proceedings of the IEEE, vol. 54, no. 9, pp. 1147-1151, September 1966.

[2] Z. Piatek and B. Baron, "Exact Closed Form Formula for Self Inductance of Conductor of Rectangular Cross Section," Progress In Electromagnetics Research M, vol. 26, pp. 225-236, 2012.

[3] M. Matsuki and A. Matsushima, "Improved Numerical Method for Computing Internal Impedance of a Rectangular Conductor and Discussions of its High Frequency Behavior," Progress In Electromagnetic Research M, vol. 23, pp. 139-152, 2012.

[4] N. Raicevic, B. Butrylo, S. Aleksic and M. Barukcic, "Improved Numerical Method for Skin Effect Computing of Conductors Having Rectangular Cross Sections," 19th International Symposium on Electromagnetic Fields in Mechatronics, Electrical and Electronic Engineering (ISEF 2019), Nancy, France, 2019, pp. 1-2, doi:10.1109/ISEF45929.2019.9096970.

[5] N. Raicevic, S. Aleksic, I. Iatcheva, M. Barukcic, "Enhanced method for pulse skin effect calculation of cylindrical conductors", COMPEL - The international journal for computation and mathematics in electrical and electronic engineering, Emerald Group Publishing Limited, vol. 39, no. 3, pp. 623-635, 2020. ISSN:0332-1649. doi:https://doi.org/10.1108/COMPEL-10-2019-0382.

[6] P. Jablonski, T. Szczegielniak, D. Kusiak and Z. Piatek, "Analytical-Numerical Solution for the Skin and Proximity Effects in Two Parallel Round Conductors," Energies, MDPI, vol. 12, no. 18, pp. 1-19 (3584), 2019. doi:10.3390/en12183584.

[7] P. Jablonski, D. Kusiak and T. Szczegielniak, "Analytical-Numerical Approach to the Skin and Proximity Effect in Lines with Round Parallel Wires," Energies, MDPI, vol. 13, no. 24, pp. 1-21 (6716), December 2020. DOI: $10.3390 /$ en 13246716.

[8] P. Rolicz, "Skin effect in a system of two rectangular conductors carrying identical currents,"Electrical Engineering, vol. 82, pp. 285-290, 2000. doi: https://doi.org/10.1007/s002020000042.

[9] A. Canova and L. Giaccone, "Numerical and Analytical Modeling of Busbar Systems, "IEEE Transaction on Power Delivery, vol. 24, no. 3, pp. 1568-1577, July 2009.

[10] M. Raven, "Experimental Measurements of the Skin Effect and Internal Inductance at Low Frequencies," Acta Technica, vol.60, pp. 51-69, 2015.

[11] R. Benato, F. Dughiero, M. Forzan, A. Paolucci, "Proximity effect and magnetic field calculation in GIL and in isolated phase bus ducts, " IEEE Transaction of Magnetics, vol. 38, pp. 781-784, 2002.

[12] H. Abedinpourshotorban, S. Mariyam Shamsuddin, Z. Beheshti, and D. N. A. Jawawi, "Electromagnetic field optimization: a physics-inspired metaheuristic optimization algorithm," Swarm and Evolutionary Computation, vol. 26, pp. 8-22, 2016.

[13] Sarajčev, P. Numerical analysis of the magnetic field of high-current bus duct and GIL systems. Energies 2011, 4 (12), 2196-2211. doi:10.3390/en4122196.

[14] D. Gerling, "Approximate analytical calculation of the skin effect in rectangular wires," in International Conference on Electrical Machines and Systems, Tokyo, 2009, pp. 1-6, doi:10.1109/ICEMS.2009.5382786. 